\title{
Convergence of Invariant Measures of Truncation Approximations to Markov Processes
}

\author{
Andrew G. Hart ${ }^{1}$, Richard L. Tweedie ${ }^{2}$ \\ ${ }^{1}$ Centro de Modelamiento Matemático, Facultad de Ciencias Físicas y Matemáticas, Universidad de Chile, Santiago, Chile \\ ${ }^{2}$ Division of Biostatistics, School of Public Health, University of Minnesota, Minneapolis, USA \\ Email: ahart@dim.uchile.cl
}

Received September 8, 2012; revised October 8, 2012; accepted October 15, 2012

\begin{abstract}
Let $Q$ be the $Q$-matrixof an irreducible, positive recurrent Markov process on a countable state space. We show that, under a number of conditions, the stationary distributions of the $n \times n$ north-west corner augmentations of $Q$ converge in total variation to the stationary distribution of the process. Twoconditions guaranteeing such convergence include exponential ergodicity and stochastic monotonicity of the process. The same also holds for processes dominated by a stochastically monotone Markov process. In addition, we shall show that finite perturbations of stochastically monotone processes may be viewed as being dominated by a stochastically monotone process, thus extending the scope of these results to a larger class of processes. Consequently, the augmentation method provides an attractive, intuitive method for approximating the stationary distributions of a large class of Markov processes on countably infinite state spaces from a finite amount of known information.
\end{abstract}

Keywords: Invariant Measure; Truncation Approximation; Augmentation; Exponential Ergodicity; Stochastic Monotonicity; Markov Process

\section{Introduction}

Let $Q=(q(i . j), i, j \in S)$ be the stable, conservative $Q$-matrix of a continuous-time Markov process on a countable state space $S=\{0,1,2, \cdots\}$ The Q-matrix satisfies

$$
\begin{aligned}
& Q(i, j) \geq 0, \text { if } j \neq i, \\
& 0 \leq q(i):=-Q(i, i)<\infty, \text { if } j=i, \text { and } \\
& \sum_{j \in S} Q(i, j)=0 \text { for all } i \in S
\end{aligned}
$$

In addition, we assume that $Q$ is regular, which means there exists no non-trivial, non-negative solution

$$
\begin{aligned}
& x=(x(j), j \in S) \text { to } \\
& \qquad \sum_{j \in S} Q(i, j) x(j)=k x(i) ; 0 \leq x(i) \leq 1, i \in S
\end{aligned}
$$

for some (and then all) $k>0$.

Under these assumptions, the state transition probabilities of the process are given by the unique $Q$-function $F=\left(F^{t}(i, j), i, j \in S, t \geq 0\right)$ which satisfies the Kol- mogorov backward equations,

$\frac{\mathrm{d}}{\mathrm{d} t} F^{t}=Q F^{t}, t \geq 0, F^{0}=I$. The object $F$, which is also called a transition function, is a family of $S \times S$ matrices indexed over the reals which constitutes an analytic semi-group. an analytic semi-group is characterised by three properties: $F^{0}$ is the identity matrix, the row sums of $F^{t}$ are less than or equal to unity and $F^{s+t}$ is equal to the matrix product $F^{s} F^{t}$ for all $s, t \geq 0$. This last property, known as the Chapman-Kolmogorov equation, implies $F^{t}=\left(F^{1}\right)^{t}$. Thus, even though $F^{t}$ is generally thought of as the matrix of state transition probabilities at time $t$, it serves as an analogue to the $t$-th power of the transition matrix of a discrete-time Markov chain on the state space $S$. Consequently, using the superscript to denote $F$ as a function of $\mathrm{t}$ should not cause any confusion. While on the subject of notation, we should mention that we are using a standard notation common in the literature of continuous-time Markov processes on general state spaces. In the discrete state space setting, this notation causes matrices to look like functions of two variables (or kernels) while measures and vectores appear to be functions over the state space. We have elected to follow this notation in an endeavour to reduce the number of subscripts and superscripts in the sequel.

Note that in the conservative setting posed here, regularity of $Q$ is equivalent to honesty and uniqueness of the transition function, that is, $\sum_{j \in S} F^{t}(i, j)=1$ for all $i \in S$ and $t \geq 0$.

The state space $S$ is irreducible if $F^{T}(i, j)>0$ for 
all $i, j \in S$ and $t \geq 0$. On such a state space, a Markov process is said to be positive recurrent or ergodic if $F^{t}(i, j) \rightarrow \pi(j)>0$ for all $i, j \in S$ as $t \rightarrow \infty$. For a positive recurrent process, it can be shown (for example, see Theorem 5.1.6 in [1]) that the $\lim \pi=\left(\pi_{i}, i \in S\right)$ satisfies

$$
\begin{aligned}
& \sum_{j \in S} \pi(i)=1 \text { and } \sum_{j \in S} \pi(i) F^{t}(i, j)=\pi(j), \\
& j \in S, t \geq 0
\end{aligned}
$$

More generally, any measure $\pi$ satisfying (1) is called an invariant or stationary measure for the process. If, in addition, the measure has mass 1 , it is referred to as a stationary or invariant distribution. Any measure satisfying (1) with " =" replaced by " $\geq$ " is called a subinvariant measure for $F$. Conversely, if $F$ has a stationary distribution $\pi$, then the process is positive recurrent and $F^{t}(i, j) \rightarrow \pi(j)$ as $t \rightarrow \infty$.

In this paper, we are interested in approximating $\pi$ using the $n \times n$ north-west corner truncations of $Q$. The analogous problem for discrete-time Markov chains has been studied in [2-7]. The final reference contains a review of the literature on the discrete-time version of the truncation problem. Some properties of truncation in continuous-time Markov processes were studied in [8,9].

Truncations of $Q$ are submatrices of $Q$ defined by ${ }_{(n)} Q=\left(_{(n)} Q(i, j), i, j \in S_{n}\right)$, where ${ }_{(n)} Q(i, j):=Q(i, j)$ and $\left(S_{n}, n \in \mathbb{N}\right)$ is an increasing sequence of subsets of S such that $\bigcup_{n \in \mathbb{N}} S_{n}=S$.

The truncation ${ }_{(n)} Q$ is not conservative. By adding the discarded transition rates to ${ }_{(n)} Q$, we may produce a conservative $Q$-matrix $\overline{{ }_{(n)} Q}$ which generates a unique, honest, finite, continuous-time Markov process. For example, we may choose to perform linear augmentation, where the aggregate of the transition rates outside of $S_{n}$ is dispersed amongst the states in $S_{n}$ according to some probability measure ${ }_{(n)} \alpha=\left({ }_{(n)} \alpha(i), i \in S_{n}\right)$. Then, the $n$-th order augmentation $\overline{{ }_{(n)} Q}$ is given by

$$
\overline{{ }_{(n)}} Q(i . j)=\left\{\begin{array}{l}
Q(i, j)+{ }_{(n)} \alpha(j) \sum_{k \notin S_{n}} Q(i, k), \text { if } i, j \in S_{n}, \\
0, \text { otherwise. }
\end{array}\right.
$$

An important example of this is where we only augment a single column, say $h$, in which case ${ }_{(n)} \alpha$ is the Dirac measure at $h$ and we obtain The $n$-th order augmentation ${ }_{(n)} Q_{h}$ as

$$
{ }_{(n)} Q(i . j)=\left\{\begin{array}{l}
Q(i, j)+\delta_{j h} \sum_{k \notin S_{n}} Q(i, k), \text { if } i, j \in S_{n}, \\
0, \text { otherwise. }
\end{array}\right.
$$

Here, $\delta_{j h}$ denotes the kronecker delta.
Linear augmentation obtains exactly one irreducible, closed class $A_{n} \subseteq S_{n}$ together with zero or more open classes from which $A_{n}$ is accessible. Since $A_{n}$ is closed, $\overline{{ }_{(n)} Q}$ is conservative on $A_{n}$ and so the minimal $\overline{(n)} Q$-function $\overline{{ }_{(n)} F}$ is honest and positive recurrent on $A_{n}$. Finiteness of $S_{n}$ ensures that the remaining open classes are transient. Hence, there exists a unique invariant measure for $\overline{{ }_{(n)} F}$. We shall be mainly concerned with ${ }_{(n)} Q_{h}$ where either $h=0$ or $h=n$. The minimal $\overline{{ }_{(n)} Q}$-function will be denoted ${ }_{(n)} F_{h}$ while ${ }_{(n)} \pi_{h}=\left(_{(n)} \pi_{h}(i), i \in S_{n}\right)$ will be its invariant probability measure.

Two obvious questions now arise. Firstly, when does

$$
{ }_{(n)} \pi_{h} \stackrel{w}{\longrightarrow} \pi \text { as } n \rightarrow \infty \text { ? }
$$

Here, we use $\stackrel{w}{\longrightarrow}$ to denote convergence in total variation norm. Secondly, how quickly does this convergence occur? This paper considers the first question. We shall present augmentation strategies for approximating invariant distributions for two classes of Markov processes via $_{(n)} \pi_{h}$ for $n$ large. The classes are:

- Markov processes which satisfy

$$
\left|F^{t}(i, j)-\pi(j)\right|=O\left(\mathrm{e}^{-a t}\right), i, j \in S, t \geq 0,
$$

for some $\alpha>0$. Such processes are called exponentially ergodic.

- Stochastically monotone Markov processes, which have the property that

$$
\sum_{j \geq n} F^{t}(i, j) \leq \sum_{j \geq n} F^{t}(k, j)
$$

for all $i \leq k, i, k, n \in S$ and $t \geq 0$, and processes dominated by stochastically monotone processes.

Parallelling results for discrete-time chains in [7], we shall also show that Markov processes constructed from finite perturbations of stochastically monotone processes are always dominated by some other stochastically monotone process. This extends the class of processes for which our results are applicable.

In the next section, we begin by showing that the limit of the ${ }_{(n)} \pi^{\prime}$ is unique when it exists. Then, Section 3 considers exponentially ergodic Markov processes while Section 4 studies stochastically monotone Markov processes and their above-mentioned variations.

Finally, some concluding remarks are made in Section 5.

\section{Preliminaries}

The problem of proving that ${ }_{(n)} \pi_{h} \stackrel{w}{\longrightarrow} \pi$ may be bro- 
ken into two parts. Firstly we must show that ${ }_{(n)} \pi_{h}$ converges weakly to some limit, say $\bar{\pi}$, and secondly, that $\bar{\pi} \equiv \pi$. We consider the latter in this section.

Theorem 2.1 Consider a sequence of linearly augmented $Q$-matrices $\left.{ }_{(n)} Q_{h}\right)$ derived from $Q$ and let ${ }_{(n)} F_{h}$ be the minimal ${ }_{(n)} Q_{h}$-function. Then

$$
{ }_{(n)} F_{h}^{t}(i, j) \mapsto F^{t}(i, j) \text { as } n \rightarrow \infty, i, j \in S \text {. }
$$

Proof: Let ${ }_{(n)} F$ denote the minimal ${ }_{(n)} Q$-function. Firstly, observe that ${ }_{(n)} F^{t}(i, j) \leq_{(n)} F_{h}^{t}(i, j)$ for all

$i, j \in S$ and $t \geq 0$. This can be seen inductively using the backward integral recurrences for ${ }_{(n)} F$ and ${ }_{(n)} F_{h}$. The argument parallels the proof of Theorem 2.2.14 in [1] which states that

$$
{ }_{(n)} F^{t}(i, j) \nearrow F^{t}(i, j) \text { as } n \rightarrow \infty
$$

for all $i, j \in S, t \geq 0$

Next, since ${ }_{(n)} F_{h}$ is honest and ${ }_{(n)} F$ is dishonest, we see that

$$
{ }_{(n)} F^{t}(i, j) \leq_{(n)} F_{h}^{t}(i, j) \leq_{(n)} F^{t}(i, j)+\delta_{n}^{t}(i)
$$

for $i, j \in S_{n}, t \geq 0, n \geq 1$, where

$$
\delta_{n}^{t}(i):=1-\sum_{k \in S_{n}}(n) F^{t}(i, k), i \in S_{n}, t \geq 0
$$

Applying (4) to (6) together with monotone convergence shows that $\delta_{n}^{t}(i)$ monotonically decreases to 0 as $n \rightarrow \infty$. Taking limits in $n$ on both sides of (5) then completes the proof.

Remark 2.2 Although we have only considered linear augmentations, the statement and proof of Theorem 2.1 is in fact valid for any sequence of augmentations $\left(\overline{{ }_{(n)} F^{t}}\right)$.

Since the transition function ${ }_{(n)} F_{h}$ is finite, it is positive recurrent on some subset of $S_{n}$. Hence it possesses a unique stationary distribution ${ }_{(n)} \pi_{h}$ and

$$
\sum_{j \in S_{n}}{ }_{(n)} \pi_{h}(i)_{(n)} F_{h}^{t}(i, j)={ }_{(n)} \pi_{h}(j)
$$

for $j \in S_{n}, t \geq 0$. Positive recurrence establishes anequivalence between the stationary distributions for ${ }_{(n)} F_{h}^{t}$ and invariant distributions for ${ }_{(n)} Q_{h}$. An invariant distribution for an arbitrary $Q$-matrix $Q$ is any probability measure $\pi$ such that $\sum_{i \in S} \pi(i) Q(i, j)=0$ for all $j \in S$. So, ${ }_{(n)} \pi_{h}$ uniquely satisfies $\sum_{i \in S_{n}(n)} \pi_{h}(i){ }_{(n)} Q_{h}(i, j)=0$ for all $j \in S_{n}$

Let us assume for the moment that ${ }_{(n)} \pi_{h}$ converges weakly to some limit measure $\bar{\pi}=(\bar{\pi}(i), j \in S)$. We require that $\bar{\pi} \equiv \pi$. Weak convergence to $\bar{\pi}$ implies that $\bar{\pi}$ is a probability distribution. By taking the limit infimum on both sides of (7) and applying Fatou's Lemma, we have

$$
\begin{aligned}
& \sum_{j \in S} \bar{\pi}(i) F^{t}(i, j) \\
\leq & \liminf _{n \rightarrow \infty} \sum_{j \in S}{ }_{(n)} \pi_{h}(i)_{(n)} F_{h}^{t}(i, j) \\
= & \liminf _{n \rightarrow \infty} \pi_{(n)}(j)=\bar{\pi}(j)
\end{aligned}
$$

for $j \in S, t \geq 0$. The measure $\bar{\pi}$ is therefore a subinvariant probability measure for $F$. However, $F$ is positive recurrent and hence, by Theorem 4 in [10], $\bar{\pi}$ is both invariant and the unique probability measure satisfying (1). Hence, $\bar{\pi} \equiv \pi$.

\section{Exponential Ergodicity}

Let $Q$ be the $Q$-matrix of a positive recurrent Markov process $\chi$ on $S$. Consider an increasing sequence of sets $\left(S_{n}, n \in \mathbb{N}\right)$ such that $\bigcup_{n \in \mathbb{N}} S_{n}=S$ and $0 \in S_{n}$ for all n. Let ${ }_{(n)} Q$ be the truncation of $Q$ corresponding to $S_{n}$. In this section, we shall consider augmentations ${ }_{(n)} Q_{0}$ obtained by linearly augmenting ${ }_{(n)} Q$ in column 0 . We shall prove that exponential ergodicity of the Markov process is sufficient for ${ }_{(n)} \pi \stackrel{w}{\longrightarrow} \pi$ as $n \rightarrow \infty$ where ${ }_{(n)} \pi$ is taken to be ${ }_{(n)} \pi_{0}$, the invariant distribution for ${ }_{(n)} Q_{0}$. In order to do this, we shall require the notion of a $V$-norm. Let $V=(V(i), i \in S)$ be an arbitrary vector (function) such that $1 \leq V(i) \leq \infty$ for all $i \in S$. In future, we abreviate this to $V \geq 1$. The $V$ norm of a signed measure $n$ is then

$$
\|V\|_{V}:=\sup _{f: f f \mid \leq V}|v(f)|=\sum_{j \in S}|v(i)| V(i)
$$

If $A=[A(i, J) i \in S, J \in B(S))$ is a matrix, then the $V$-norm of $A$ is

$$
\|A\|_{V}:=\sup _{i \in S} \frac{\|A(i, \cdot)\|_{V}}{V(i)}
$$

Rather than working with the $Q$-matrix augmentationsdirectly, we will use the $\beta$-resolvents associated with these. The $\beta$-resolvent of a continuoust-time Markov process is the stochastic matrix $R_{\beta}=\left(R_{\beta}(i, j), i, j \in S\right)$ given by, $R_{\beta}(i, j):=\int_{0}^{\infty} \beta \mathrm{e}^{-\beta t} F^{t}(i . j) \mathrm{d} t, i, j \in S, \beta>0$. We note that $R_{\beta}$ satisfies the resolvent forms of both the backward and forward equations which are $R_{\beta}=I+\beta^{-1} Q R_{\beta}$ and $R_{\beta}=I+\beta^{-1} R_{\beta} Q$ respectively.

Since $Q$ is regular, $R_{\beta}$ is the unique solution to the resolvent form of the backward equations. Let ${ }_{(n)} R_{\beta}$ and ${ }_{(n)} R_{\beta, h}$ denote the unique $\beta$-resolvents of ${ }_{(n)}^{(n)} F$ and ${ }_{(n)} F_{h}$ respectively. Here, ${ }_{(n)} F$ is the minimal ${ }_{(n)} Q$-funcction while ${ }_{(n)} F_{h}$ denotes the minimal ${ }_{(n)} Q_{h}$ function. Since $S_{n}$ is a finite set, ${ }_{(n)} Q_{h},{ }_{(n)} F_{h}$ and 
${ }_{(n)} R_{\beta, h}$ have the same invariant distribution ${ }_{(n)} \pi_{h}$. The same is true for $F$ and $R_{\beta}$, which share the distribution $\pi$.

In the sequel, we shall have need of the following corollary to Theorem 2.1.

\section{Corollary 3.1}

i. For all $i, j \in S_{n}, \beta>0$ and $n \in \mathbb{N}$,

$$
\begin{aligned}
{ }_{(n)} R_{\beta}(i, j) & \leq{ }_{(n)} R_{\beta, h}(i, j) \\
& \leq{ }_{(n)} R_{\beta}(i, j)+\rho_{\beta, n}(i)
\end{aligned}
$$

where $\rho_{\beta, n}(i):=1-\sum_{j \in S_{n}(n)} R_{\beta}(i, j)$; and

ii. ${ }_{(n)} R_{\beta, h}(i, j) \rightarrow R_{\beta}(i, j)$ as $n \rightarrow \infty$.

Proof: Part $i$ is obtained by integrating both sides of (5) with respect to be $\beta \mathrm{e}^{-\beta t} \mathrm{~d} t$. Part ii then follows by taking limits in (8) and observing that

${ }_{(n)} R_{\beta}(i, j) \nearrow R_{\beta}(i, j)$ and $\rho_{\beta, n}(i) \searrow 0$ as $n \rightarrow \infty$

Next, the various "drift to $C$ " conditions introduced in [11] will play an important role in allowing us to pass between the continuous-time process and the discretetime $\beta$-resolvent chain. The drift conditions require the notion of a petite set in both continuoustime processes and discrete-time chains. Let $\mathcal{B}(S)$ denote the Borel $\sigma$-algebra on $S$. Then, A set $C \in \mathcal{B}(S)$ is a petite set in the continuous-time setting if there exists a probability distribution $a$ on $\left(R^{+}, \mathcal{B}\left(R^{+}\right)\right)$and a non-trivial positive measure $\mathrm{v}_{a}$ such that

$$
K_{a}(i, J):=\int_{0}^{\infty} F^{t}(i, J) a(\mathrm{~d} t) \geq V_{a}(J)
$$

for $i \in C, J \in S(\mathcal{B})$, where $F^{t}(i, J) \sum_{j \in J} F^{t}(i, j)$.

Petite sets for discrete-time chains are defined analogously. According to Theorem 5.1 in [11], the following three drift conditions are equivalent, although the petite set $\mathrm{C}$ and function $\mathrm{V}$ may differ in each instance.

$\left(\mathcal{D}_{T}\right)$ : Drift for $\mathrm{T}$-skeletons. For some $T>0$, there exist constants $\lambda(S)$ bounded for all $s \in(0, T]$ with $\lambda(T)<1, b<\infty$, together with a petite set $C \in \mathcal{B}(S)$ and a function $V_{T} \geq 1$ such that

$$
\sum_{j \in S} F^{S}(i, j) V_{T}(j) \leq \lambda(s) V_{T}(i)+b \mathbb{I}_{C}(i),
$$

for $i \in S, s \leq T$. We use $\mathbb{I}_{C}(i)$ to denote the indicator function of the set $C$ which is 1 if $i \in C$ and 0 otherwise.

$\left(\breve{\mathcal{D}}_{\beta}\right)$ : Drift for $\beta$-resolvents. For some $\lambda \in(0,1), b<\infty, \beta>0$, a petite set $C \in \mathcal{B}(S)$ and a function

$$
\sum_{j \in S} R_{\beta}(i, j) V_{\beta}(j) \leq \lambda V_{\beta}(i)+b \mathbb{I}_{C}(i), i \in S .
$$

$(\tilde{\mathcal{D}})$ : Drift for the $Q$-matrix. For constants $b, c>0$, a petite set $C \in \mathcal{B}(S)$ and a function $\tilde{V} \geq 1$, $\sum_{j \in S} q(i, j) \tilde{V}(j) \leq-c \tilde{V}(i)+b \mathbb{I}_{C}(i), i \in S$.

An irreducible continuous-time Markov process $\mathrm{X}$ is $V$-uniformly ergodic if, for some invariant probability kernel $\pi,\left\|F^{t}-\pi\right\|_{V} \rightarrow 0$ as $t \rightarrow \infty$. In the special case where $V \equiv 1$, the chain is said to be uniformly ergodic or strongly ergodic: For all $i \in S,\left\|F^{t}-\pi\right\|_{1} \rightarrow 0$ as $t \rightarrow \infty$ where, by an abuse of notation, we use $\pi$ to denote the invariant transition kervnel $\pi(i, j)=\pi(j)$ for all $i, j \in S$.

The following theorem collects together a number of results on exponential and $V$-uniform ergodicity of Markov processes from the literature.

Theorem 3.2 Let $X$ be an irreducible, aperiodic continuous-time Markov process on S. The following conditions are equivalent.

i. One of the drift conditions $\left(\mathcal{D}_{T}\right),\left(\breve{\mathcal{D}}_{\beta}\right)$ or $(\tilde{\mathcal{D}})$ holds, in which case they all hold, but not necessarily with the same petite set $C$;

ii. For all $T>0$, the $T$-skeleton chain is geometrically ergodic;

iii. For all $\beta>0$, the $\beta$-resolvent chain is geometrically ergodic;

iv. $\mathrm{X}$ is exponentially ergodic.

v. $\mathrm{X}$ is $V$-uniformly ergodic for some $V \geq 1$.

In particular, it is $V_{T}$-uniformly ergodic, $V_{\beta}$-uniformly ergodic and $\tilde{V}$-uniformly ergodic where $V_{T}, V_{\beta}$ and $\tilde{V}$ satisfy $\left(\mathcal{D}_{T}\right),\left(\breve{\mathcal{D}}_{\beta}\right)$ and $(\tilde{\mathcal{D}})$ respectively.

\section{Proof:}

ii $\Leftrightarrow$ iii $\Leftrightarrow$ iv. This was proved in Theorem 5.3 of [11]. $\mathrm{i} \Leftrightarrow$ iv. Theorem 5.1 of [11] shows that

$\left(\mathcal{D}_{T}\right),\left(\breve{\mathcal{D}}_{\beta}\right)$ and $(\tilde{\mathcal{D}})$ satisfy a solidarity property in that either all of them hold or none hold. Next, fix $N \geq 1$ and set $C=\{1,2, \cdots, N\}$ which is trivialy petite Since it is finite. In $(\tilde{\mathcal{D}})$, set $c=\lambda$ and $V(i)=c y_{i}+1$, where $N, \lambda$ and the $y_{i}$ 's are those appearing in Theorem 3 of [12]. Finally, an appropriate relabelling of the states in $S$ reveals $(\tilde{\mathcal{D}})$ to be equivalent to the necessary and sufficient condition for exponential ergodicity givenin Part (ii) of Theorem 3 in [12]. Consequently $\mathrm{X}$ is exponentially ergodic if and only if $(\tilde{\mathcal{D}})$ orany of the other drift criteria holds.

$\mathrm{i} \Rightarrow \mathrm{v}$. Theorem 5.2 in [11] says that any of $\left(\mathcal{D}_{T}\right),\left(\breve{\mathcal{D}}_{\beta}\right)$ or $(\tilde{\mathcal{D}})$ is sufficient for $\mathrm{X}$ to be $V$-uniformly ergodic where $V$ is either $V_{T}, V_{\beta}$ or $\tilde{V}$ respectively.

$\mathrm{v} \Rightarrow$ ii. If $\mathrm{X}$ is $V$-uniformly ergodic for some $V \geq 1$, then so to is the $T$ - skeleton for any $T>0$ and an application of Theorem 16.0.1 in [13] shows that $\left\|F^{T n}-\pi \mid\right\|_{V} \leq M \rho^{n}$ for some $M<\infty$ and $\rho \in(0,1)$. 
Geometric ergodicity of the $T$-skeleton $F^{T n}$ then follows from the definition of the $V$-norm.

Next, suppose that the Markov process $\mathrm{X}$ is exponenttially ergodic. From Theorem 3.2, there exist constants $0<c, d<\infty$ and a function $V \geq 1$ such that

$$
\begin{aligned}
& \sum_{j \in S} Q(i, j) V(j) \leq-c V(i)+d \mathbb{I}_{C}(i), \\
& i \in S .
\end{aligned}
$$

Without loss of generality, we may take $C=\{0\}$ and assume that $V(0)=1$. The state space can always be relabelled to accommodate this convention. Then, since $V(k) \geq V(0)=1$ for all $k \neq 0$, the augmented $Q$-matrices $\left.{ }_{\left({ }_{(n)}\right.} Q_{0}\right)$ each satisfy

$$
\begin{aligned}
& \sum_{j \in S_{n}}{ }_{(n)} Q_{0}(i, j) V(j) \leq-c V(i)+d \mathbb{I}_{0}(i), \\
& i \in S_{n} .
\end{aligned}
$$

Multiplying both sides by ${ }_{(n)} R_{\beta, 0}$ and re-arranging, we obtain

$$
\begin{aligned}
& \sum_{j \in S_{n}}{ }_{(n)} R_{\beta, 0}(i, j) V(j) \\
& \leq \frac{\beta}{\beta+c} V(i)+\frac{d}{\beta+c}\left({ }_{(n)} R_{\beta, 0}(i, 0), .\right. \\
& \text { for } i \in S_{n}
\end{aligned}
$$

Now, choose $\varepsilon<c /(\beta+c)$ such that ${ }_{(N)} R_{\beta}(0,0) \geq \delta:=\varepsilon(\beta+c) / d>0$ for some

$N=N(\beta, \varepsilon) \in \mathbb{N}$. This is always possible since $R_{\beta}$ is a strictly positive matrix (in particular, $R_{\beta}(0,0)>0$ and, as noted in the proof of Corollary 3.1,

${ }_{(n)} R_{\beta}(0,0) \nearrow R_{\beta}(0,0)$ as $n \rightarrow \infty$. Therefore,

${ }_{(n)} R_{\beta, 0}(0,0) \geq{ }_{(n)} R_{\beta}(0,0) \geq \delta$. So, in addition to $R_{\beta}$

being strongly aperiodic, we see that ${ }_{(n)} R_{\beta, 0}$ is strongly aperiodic for all $n \geq N$. A transition matrix is strongly aperiodic if it is primative and possesses a non-zero diagonal entry.

Define $C_{n}:=\left\{k \in S_{n}:_{(n)} R_{\beta, 0}(k, 0) \geq \delta\right\}$. By Proposition 5.5.4 in [13], the set $C_{n}$ is petite since the singleton set $\{0\}$ is trivially petite and $\{0\}$ is uniformly accessible from $C_{n}$ under the resolvent chain ${ }_{(n)} R_{\beta, 0}$, that is, ${ }_{(n)} R_{\beta, 0}(k, 0)$ is bounded away from 0 for all $k \in C_{n}$. By the definition of $C_{n}$, we have ${ }_{(n)} R_{\beta, 0}(i, 0)<\delta$ for $i \in S_{n} \backslash C_{n}$. On the other hand, ${ }_{(n)} R_{\beta, 0}(i, 0) \leq 1$ for $i \in C_{n}$, since ${ }_{(n)} R_{\beta, 0}$ is a stochastic matrix. Hence we have

$$
\sum_{j \in S_{n}}{ }_{(n)} R_{\beta, 0}(i, j) V(j)
$$

$$
\begin{aligned}
& \leq \frac{\beta}{\beta+c} V(i)+\frac{d}{\beta+c}{ }_{(n)} R_{\beta, 0}(i, 0) \\
& \leq \frac{\beta}{\beta+c} V(i)+\frac{d}{\beta+c} \mathbb{I}_{C_{n}}(i)+\varepsilon \mathbb{I}_{S_{n} \backslash C_{n}}(i) \\
& \leq \frac{\beta}{\beta+c} V(i)+\frac{d}{\beta+c} \mathbb{I}_{C_{n}}(i)+\varepsilon V(i) \\
& =\lambda^{\prime} V(i)+b^{\prime} \mathbb{I}_{C_{n}}(i), i \in S_{n}, n \geq N,
\end{aligned}
$$

where $\lambda^{\prime}:=\beta /(\beta+c)+\varepsilon<1$ and $b^{\prime}:=d /(\beta+c)>0$.

Note that $0 \in C_{n}$ for all $n$ large enough.

Next, set $P \equiv{ }_{(n)} R_{\beta, 0}, S=S_{n}, C=C_{n}, \alpha=\{0\}, \lambda_{C}=\lambda^{\prime}$ and $b_{C}=b_{\alpha}^{*}=b_{C}^{*}=b^{\prime}$ in Theorem 6.1 of [14]. It can be seen that the conditions of the theorem are satisfied and so there exists some $V^{\prime} \geq 1$ such that

$$
\sum_{j \in S_{n}}(n) r_{\beta, 0}(i, j) V^{\prime}(j) \leq \lambda V^{\prime}(i)+b \mathbb{I}_{0}(i), i \in S_{n},
$$

where $\lambda=\frac{\lambda^{\prime}+b^{\prime} / \delta}{1+b^{\prime} / \delta}=\frac{\beta+\varepsilon(\beta+c)+d / \delta}{\beta+c+d / \delta}$ and $b=(1+1 / \delta) b^{\prime}=\mathrm{d}(1+1 / \delta) /(\beta+c)$. Furthermore, we have

$$
\left\|(n) R_{\beta, 0}(i, \cdot){ }_{(n)} \pi_{0}(\cdot)\right\|_{V} \leq M V(i) \rho^{m}, m \in \mathbb{N},
$$

where ${ }_{(n)} \pi_{0}$ is the unique invariant distribution for ${ }_{(n)} Q_{0}$, and $M<\infty$ and $\rho<1$ are completely determined by $\beta, c, d$ and $\delta$. Note that this is true for every $n \geq N$ so that the rate of convergence $\rho$ is independent of the truncation size. In addition, by applying the preceding argument directly to $Q$ instead of ${ }_{(n)} Q_{0}$, we also have $\left\|R_{\beta}^{m}(i, \cdot)-\pi(\cdot)\right\|_{V} \leq M V(i) \rho^{m}$ for all $m$, since, by assumption, (9) holds and $R_{\beta}(0,0) \geq \delta>0$. Thus, not only are $R_{\beta}$ and ${ }_{(n)} R_{\beta, 0} \quad V$-uniformly ergodic, they are geometrically ergodic with the same convergence rate $\rho$.

We can now prove the main result of this section.

Theorem 3.3 Let X be an exponentially ergodic, continuous-time Markov chain on a countable, irreducible state space $S$. Let $\pi$ and ${ }_{(n)} \pi_{0}$ be the invariant distributions for $Q$ and ${ }_{(n)} Q_{0}$ respectively. Then, $\left\|(n) \pi_{0}-\pi\right\| \rightarrow 0$ as $n \rightarrow \infty$.

Proof: Choose an arbitrary number $m \geq 2$. From the triangle inequality, we have

$$
\begin{aligned}
& \left\|(n) \pi_{0}-\pi\right\| \\
& \leq\left\|R_{\beta}^{m}(i, \cdot)-\pi\right\|+\left\|{ }_{(n)} R_{\beta, 0}^{m}(i, \cdot)-{ }_{(n)} \pi_{0}\right\| \\
& +\left\|R_{\beta}^{m}(i, \cdot)-{ }_{(n)} R_{\beta, 0}^{m}(i, \cdot)\right\| \\
& \leq 2 M V(i) \rho^{m}+\left\|R_{\beta}^{m}(i, \cdot)-_{(n)} R_{\beta, 0}^{m}(i, \cdot)\right\| .
\end{aligned}
$$

As was pointed out in [7], if $A$ and $B$ are two sto- 
chastic matrices, then

$$
\begin{aligned}
& \left\|A^{m}(i, \cdot)-B^{m}(i, \cdot)\right\| \\
& \leq \sum_{0 \leq s \leq m-1, w \in S} A^{S}(i, w)\|A(w, \cdot)-B(w, \cdot)\|,
\end{aligned}
$$

for $m \geq 2$. Applying this to the last term in (13), we obtain

$$
\begin{aligned}
& \left\|R_{\beta}^{m}(i, \cdot)-{ }_{(n)} R_{\beta, 0}^{m}(i, \cdot)\right\| \\
& \leq \sum_{0 \leq s \leq m-1, w \in S} R_{\beta}^{s}(i, w) \delta_{n}(w),
\end{aligned}
$$

where

$$
\begin{aligned}
& \delta_{n}(i):=\left\|R_{\beta}(i, \cdot)-{ }_{(n)} R_{\beta, 0}(i, \cdot)\right\| \\
& =\sum_{j \in S}\left|R_{\beta}(i, j)-{ }_{(n)} R_{\beta, 0}(i, j)\right| .
\end{aligned}
$$

Now, since $\delta_{n}(i) \rightarrow 0$ as $n \rightarrow \infty$ for all $j \in S$, we can use dominated convergence to conclude that the third term in (13) vanishes as $n$ tends to infinity. Thus,

$$
\lim _{n \rightarrow \infty}\left\|(n) \pi_{0}-\pi\right\| \leq 2 M V(i) \rho^{m}
$$

for $m \in \mathbb{N}$, and since $m$ was chosen arbitrarily,

$$
\lim _{n \rightarrow \infty}\left\|(n) \pi_{0}-\pi\right\|=0
$$

\section{Example}

Let $\lambda, \mu>0$ and define $Q$ by

$$
Q(i, j)=\left\{\begin{array}{l}
\lambda, \text { if } i \geq 0, j=i+1, \\
\mu, \text { if } j=0 \text { and } i>1, \\
\lambda, \text { if } j=i=0 \\
-(\lambda+\mu), \text { if } j=i>0 \\
0, \text { otherwise }
\end{array}\right.
$$

The process with this $Q$-matrix is essentially a renewal process with renewal times marked by visits to state 0 . Each renewal time consists of a geometric number of exponential times of mean $1 / \lambda$ followed by an exponential time of mean $1 / \mu$. At each jump, the process passes from state $i$ to state $i+1$ with probability $\gamma=\lambda /(\lambda+\mu)$ and falls back to state 0 with probability $1-\gamma$. the state space is clearly irreducible and the process has a geometric stationary distribution $\pi$, where $\pi(i)=(1-\gamma) \gamma^{i}$. Existence of the stationary distribution ensures positive recurrence.

Next, let the vector $\tilde{V}$ be given by $\tilde{V}(i)=(1+\delta)^{i}$, where $\delta \in(0, \mu / \lambda)$. Also define $b=\mu$ and Set $C=\left\{i \in S: \mu-(\lambda \delta-\mu)(1+\delta)^{i}>-C\right\}$, where $c$ is a small positive number. Then, the drift condition $(\tilde{D})$ holds for the specified $\tilde{V}, C, b$ and $c$. The process is therefore exponentially ergodic by Theorem 3.2. Further, all the conditions of Theorem 3.3 are satisfied. Thus, we can construct augmentations ${ }_{(n)} Q_{0}$ on corresponding sets $S_{n}=\{0,1, \cdots, n\}$ and use their invariant distributions ${ }_{(n)} \pi_{0}$ to approximate $\pi$.

We can confirm this by solving

$\sum_{i=0(n)}^{n} \pi_{0}(i)_{(n)} Q_{0}(i, j)=0, j=0,1, \cdots, n$ with

$\sum_{i=0(n)}^{n} \pi_{0}(i)=1$. We have ${ }_{(n)} \pi_{0}(i)=(1-\gamma) \gamma^{i} /\left(1-\gamma^{n}\right)$

for $i=0,1, \cdots, n$, from which it is evident that ${ }_{(n)} \pi_{0}(i) \searrow \pi(i)$ as $n \rightarrow \infty$. Convergence in total variation follows by the same argument used later in the proof of Theorem 4.2 .

\section{Stochastic Monotonicity}

In this section, we develop results for stochastically monotone Markov processes. Our key result says that stochastic monotonicity of the process is sufficient for (2) to hold under arbitrary linear augmentation. The remaining results extend this to larger classes of Markov processes. While our methods generally parallel those employed in [6] TO study the same problem in discrete-time Markov chains, itt is necessary to take greater care constructing the augmentations in the continuous-time setting.

Let $\mu$ and $v$ be two non-trivial measures. Then, $v$ stochastically dominates $\mu$ if $\sum_{k \geq n} \mu(k) \leq \sum_{k \geq n} v(k)$ for all $n \in \mathbb{N}$, in which case we write $\mu \preceq v$. If $F$ and $\tilde{F}$ are two transition functions, we say that $\tilde{F}$ stochastically dominates $F$ (written $F \preceq \tilde{F}$ ) if, for all $t \geq 0, \quad F^{t}(i, \cdot) \tilde{F}^{t}(i, \cdot)$ for all $i \in S$. A more strict classification is stochastic comparability. The transition functions $F$ and $\tilde{F}$ are stochastically comparable if $F^{t}(i, \cdot) \preceq \tilde{F}^{t}(m, \cdot)$ for all $t \geq 0$ and $i, m \in S$ with $i \leq m$. We use the notation $F \sqsubseteq \tilde{F}$ to mean that $F$ and $\tilde{F}$ are stochastically comparable. A stochastically monotone Markov process is one whose transition function is stochastically comparable to itself. Thus, if $F$ is stochastically dominated by a transition function $\tilde{F}$ which itself is stochastically monotone, then $F$ and $\tilde{F}$ are stochasticallly comparable. Clearly, $F \sqsubseteq \tilde{F}$ implies $F \preceq \tilde{F}$.

The following theorem is the key to obtaining sufficient conditions for (2) to hold in continuous time. It characterises stochastic comparability and monotonicity in terms of $Q$-matrix structure and is a special case of a more general result which was proved in [15] (also see Theorem 7.3.4 in [1] for an account). The reader is directed to the last two citations for the proof.

Theorem 4.1 ([15] and [1, Chapter 7.3])

i. Let $Q$ and $\tilde{Q}$ be two conservative $Q$-matrices. Their corresponding minimal transition functions $F$ 
and $\tilde{F}$ are stochastically comparable iff, whenever $i \leq m$, and $\mathrm{k}$ is such that either $k \leq i$ or $k>m$, then

$$
\sum_{j \geq k} Q(i, j) \leq \sum_{j \geq k} \tilde{Q}(m, j) .
$$

ii. Let $Q$ be a conservative $Q$-matrix. Its minimal $Q$-function $F$ is stochastically monotone iff, Whenever $i \leq m$ and $\mathrm{k}$ is such that either $k \leq i$ or $k>m$, then

$$
\sum_{j \geq k} Q(i, j) \leq \sum_{j \geq k} Q(m, j) \text {. }
$$

As a consequence of this result, we shall speak of stochastically monotone $Q$-matrices and of two $Q$-matrices as being stochastically comparable, etc. This abuse of terminology should not cause any confusion.

If $\tilde{F}$ is an irreducible, positive recurrent transition function and it stochastically dominates another irreducible transition function $F$, then $F$ is also positive recurrent. Furthermore, if $\pi$ and $\tilde{\pi}$ denote the stationary distributions of $F$ and $\tilde{F}$ respectively, then $\pi \preceq \tilde{\pi}$, which can be seen by letting $t \rightarrow \infty$ in

$$
\sum_{j \geq k} F^{t}(i, j) \leq \sum_{j \geq k} \tilde{F}^{t}(i, j), \forall i, k \in S .
$$

In fact, we may say something stronger than this. If $F$ is reducible and contains a collection of closed ireducible classes $\left(C_{i}\right)$, each $C_{i}$ is positive recurrent with invariant probability measure $\pi_{i}$. Since $F$ is dominated by $\tilde{F}$ on $C_{i}$, it follows that $\pi_{i} \preceq \tilde{\pi}$. Now, any invariant measure on $S$ for $Q$ can be written as a linear combination of the $\pi_{i}$ 's; that is, $\pi=\sum_{i} a(i) \pi_{i}$ for some probability measure $a=(a(i), i=1,2, \cdots)$. Therefore, $\pi \preceq \tilde{\pi}$ for all invariant distributions $\pi$.

Throughout the rest of this section, we shall use the north-west corner truncations of $Q$, that is, truncations of the form $S_{n}=\{0,1,2, \cdots, n\}$ for $n=0,1,2, \cdots$

\subsection{Stochastically Monotone Processes}

Let $Q$ be the $Q$-matrix of a positive recurrent, stochastically monotone Markov process $F$. By construction, the $n \times n$ north-west corner truncations of $Q$ augmented in the nth column are stochastically monotone. Since ${ }_{(n)} Q_{n}$ is conservative on a finite set $S_{n}, \quad{ }_{(n)} F_{n}$ has precisely one positive recurrent class, which contains $n$ and is a subset of or equal to $S_{n}$. Its limiting distribution ${ }_{(n)} \pi_{n}$ satisfies $\sum_{i \in S_{n}(n)} \pi_{n}(i)_{(n)} Q_{n} \pi(i, j)=0$

for all $j \in S$. From Theorem 4.1, we also see that ${ }_{(n)} F_{n}$ is stochastically monotone.

Let $\overline{{ }_{(n)} Q}$ be an arbitrary augmentation of ${ }_{(n)} Q$ and note that $\overline{{ }_{(n)} Q}$ is stochastically comparable with ${ }_{(n)} Q_{n}$. As per our comments above, the minimal $\overline{{ }_{(n)} Q}$-function $\overline{(n)} F$ is positive recurrent on one or more irreducible subsets of $S_{n}$ and hence any invariant distribution for $\overline{{ }_{(n)}} Q$, say ${ }_{(n)} \pi$, is stochastically dominated by ${ }_{(n)} \pi_{n}$. Now, let us extend ${ }_{(n)} Q_{n}$ and $\overline{{ }_{(n)} Q}$ to $S$ as follows:

$$
{ }_{(n)} Q_{n}(i, j)=\left\{\begin{array}{l}
Q(i, j), \text { if } j<n, \\
\sum_{k \geq n} Q(i, k), \text { if } j=n \text { and } i \leq n, \\
\sum_{k=n}^{i-1} Q(i, k), \text { if } i>n \text { and } j=n, \\
\sum_{k \geq i} Q(i, k), \text { if } i>n \text { and } j=i, \\
0, \text { otherwise, }
\end{array}\right.
$$

and

$$
\overline{{ }_{(n)} Q}(i, j)=\left\{\begin{array}{l}
\overline{{ }_{(n)} Q}(i, j), \text { if } 0 \leq i, j \leq n, \\
\sum_{k=n}^{i-1} Q(i, k), \text { if } i>n \text { and } j=0, \\
\sum_{k \geq i} Q(i, k), \text { if } i>n \text { and } j=i, \\
0, \text { otherwise. }
\end{array}\right.
$$

We also extend ${ }_{(n)} \pi_{n}$ and ${ }_{(n)} \pi$ to $S$ by appending a countably infinite number of 0 's to each, so that ${ }_{(n)} \pi_{n}(i)={ }_{(n)} \pi(i)=0$ for all $i>n$. Note that ${ }_{(n)} \pi_{n}$ (resp. $\left._{(n)} \pi\right)$ remains invariant for ${ }_{(n)} Q_{n}$ (resp. $\overline{{ }_{(n)} Q}$ ).

Moreover, since the minimal ${ }_{(n)} Q_{n}$-function ${ }_{(n)} F_{n}$ is positive recurrent on some subset of $\{0,1, \cdots, n\}$ containing $n$ and transient elsewhere in $S$, the measure ${ }_{(n)} \pi_{n}$ is the limiting distribution of

${ }_{(n)} F_{n}:{ }_{(n)} F_{n}^{t}(i, j) \rightarrow{ }_{(n)} \pi_{n}(j)$ as $t \rightarrow \infty$ for all

$i, j \in S$. Similarly, ${ }_{(n)} \pi$ is the limiting distribution for the minimal $\overline{{ }_{(n)} Q}$-function $\overline{{ }_{(n)} F}$ when given an appropriate initial distribution.

The stochastically monotone matrix ${ }_{(n)} Q_{n}$ dominates $\overline{{ }_{(n)} Q}$ while ${ }_{(n)} Q_{n}$ and ${ }_{(n+1)} Q_{n+1}$ are stochastically comparable for all $n=0,1,2, \cdots$ So too are ${ }_{(n)} Q_{n}$ and $Q$ for all $n$. Thus, $\overline{(n)} Q \sqsubseteq{ }_{(n)} Q_{n} \sqsubseteq_{(n+1)} Q_{n+1} \sqsubseteq Q$. An application of Part $\mathrm{i}$ of Theorem 4.1 then shows that $\overline{{ }_{(n)} F} \sqsubseteq{ }_{(n)} F_{n} \sqsubseteq{ }_{(n+1)} F_{n+1} \sqsubseteq F$ for all $n \in \mathbb{N}$. Consquently,

$$
\overline{{ }_{(n)} \pi} \preceq{ }_{(n)} \pi_{n} \preceq_{(n+1)} \pi_{n+1} \preceq \pi,
$$

where $\pi$ is the unique stationary distribution for $F$. The sequence $\left.{ }_{(n)} \pi\right)$ is therefore tight and so ${ }_{(n)} \pi(j) \rightarrow \pi_{j}$ for all $j \in S$ as $n \rightarrow \infty$. The same is true for $\left.{ }_{(n)} \pi_{n}\right)$.

From (19), we observe that 
$0 \leq \sum_{i \geq k}\left(\pi(i)-{ }_{(n)} \pi_{n}(i)\right) \leq \sum_{i \geq k}\left(\pi(i)-{ }_{(n)} \pi(i)\right)$, for all $k \in S$, and so ${ }_{(n)} \pi_{n}$ is at least as good an approximation to $\mathrm{p}$ as ${ }_{(n)} \pi$. Thus, any invariant measure derived from a north-west corner truncation of $Q$ augmented in its last column is optimal for approximating $\pi$.

As was pointed out in [7], the pointwise convergence of measures on a countable set can easily be extended to convergence in total variation. We therefore have the following result.

Theorem 4.2 Let $Q$ be the $Q$-matrix of a positive recurrent, stochastically monotone Markov process on $S$. Let $\mathrm{p}$ be the stationary distribution of the minimal $Q$-function $F$ and denote the invariant distribution of an arbitrary $n \times n$ north-west corner augmentation

$\overline{ }_{(n)} Q$ by ${ }_{(n)} \pi$. Furthermore, let ${ }_{(n)} Q_{n}$ be the $n \times n$ north-west corner truncation augmented in column $n$ and take ${ }_{(n)} \pi_{n}$ to be its invariant distribution. Then,

$\|(n) \pi-\pi\| \rightarrow 0$ as $n \rightarrow \infty$. The same is true of the sequence $\left({ }_{(n)} \pi_{n}\right)$ which is the optimal approximation in the sense that its tail mass more closely approximates that of $\pi$.

Proof: The fact that, for all $j \in S,{ }_{(n)} \pi(j) \rightarrow \pi(j)$ and ${ }_{(n)} \pi_{n}(j) \rightarrow \pi(j)$ as $n \rightarrow \infty$ was established in the preceding discussion. So too was the optimality of ${ }_{(n)} \pi_{n}$ as an approximation to $\pi$. To prove convergence in total variation, fix an arbitrary finite $k \in S$. Then, we obtain

$$
\begin{aligned}
\left\|{ }_{(n)} \pi-\pi\right\| & =\left.\sum_{j \in S}\right|_{(n)} \pi(j)-\pi(j) \mid \\
& \leq\left.\sum_{j \leq k}\right|_{(n)} \pi(j)-\pi(j)\left|+\sum_{j>k}\right|_{(n)} \pi(j)+\pi(j) \mid \\
& \leq\left.\sum_{j \leq k}\right|_{(n)} \pi(j)-\pi(j) \mid+2 \sum_{j>k} \pi(j) .
\end{aligned}
$$

The analogous statement holds for ${ }_{(n)} Q_{n}$ and the proof is completed by letting first $n$ and then $k$ tend to infinity.

As remarked in [6], ${ }_{(n)} \pi(a)$ will be strictly positive for sufficiently large $\mathrm{n}$ where $\mathrm{a}$ is an arbitrary state in $S$. Thus, $S_{n}$ contains a positive recurrent class to which $n$ belongs. Computationally speaking, this means that any invariant distribution will suffice as an approximation to $\pi$, provided $n$ is sufficiently large.

Finally, if $n$ is large enough so that ${ }_{(n)} Q$ possesses a quasistationary distribution (n)r supported on a nondegenerate irreducible subset of $S_{n}$, then the sequence of distributions $\left.{ }_{\left({ }_{(n)}\right.} \gamma\right)$ converges weakly to $\pi$. We can always find a sequence $\left(C_{n}, n=1,2, \cdots\right)$ of irreducible sets such that $C_{1} \subseteq C_{2} \subseteq \cdots \subseteq S$ and $\bigcup_{n \in \mathbb{N}} C_{n}=S$.

See Lemma 5.1 in [16] for a proof of this; the ana- logue for discrete-time Markov chains may be found in [3], Theorem 3.1.

For a finite state Markov process, every quasistationary distribution is equivalent to a probabilitynormalised left eigenvector of its $Q$-matrix restricted to an ireducible class. In other words, If $Q$ is a nonconservative $Q$-matrix on a finite state space $\mathrm{S}$ containing an ireducible class $C \in S, r=(r(i), i \in C)$ is a quasistationary distribution on $\mathrm{C}$ for the process if and only if $\sum_{i \in C} r(i)=1$ and, for some $\lambda>0$,

$$
\sum_{j \in S} r(i) Q(i, j)=-\lambda r(j), j \in C .
$$

Note that by virtue of $\lambda$-theory, $r(i)$ is strictly positive for all $i \in C$. By convention, we extend $r$ to $S$ by setting $r(i)=0$ for $i \in S \backslash C$. If we then construct the linear augmentation $\bar{Q}$ as

$$
\bar{Q}(i, j)=Q(i, j)-\left(\sum_{k \in C} Q(i, k)\right) r(j),
$$

$i, j \in S$, it is not difficult to see that the invariant distribution $\pi$ for $\bar{Q}$ is unique and equivalent to $r$.

Now, let us return to the case of a countably infinite state space. Given $n$ large, we may construct $\overline{{ }_{(n)} Q}$ from ${ }_{(n)} Q$ in the same manner as (20) using a left eigenvector ${ }_{(n)} r$ of ${ }_{(n)} Q$ supported on an irreducible class $C_{n}$. The conditions of Theorem 4.2 are satisfied and so the sequence $\left.{ }_{(n)} r\right)$ converges in total variation to the invariant distribution of $Q$. This observation subsumes results concerning the truncation approximation of invariant distributions of birth-death processes and subcritical Markov branching processes, for example, see [16-18]. The convergence of quasistationary distributions of truncations to the invariant distribution of the original process also holds under the weaker conditions we discuss in the next two subsections.

\subsection{Processes Dominated by Stochastically Monotone Processes}

Now we shall consider a much larger class of Markov processes, namely those whose transition functions are stochastically dominated by a positive recurrent, stochastically monotone process. To begin, let $\tilde{F}$ be the stochastically monotone transition function of an irreducible, positive recurrent Markov process. Suppose that $\tilde{F}$ dominates a transition function $F$. We shall use $Q$ and $\tilde{Q}$ to denote the corresponding $Q$-matrices. As noted earlier, $F$ must be positive recurrent and the invariant distributions $\pi$ and $\tilde{\pi}$, corresponding to $F$ and $\tilde{F}$ respectively, satisfy $\pi \preceq \tilde{\pi}$.

Let ${ }_{(n)} Q_{n}$ and ${ }_{(n)} \tilde{Q}_{n}$ respectively denote the $n \times n$ 
north-west corner truncations of $Q$ and $\tilde{Q}$ augmented in the $n$th column. By extending these in the analogous way to (18) and applying Part i of Theorem 4.1, we see that ${ }_{(n)} F_{n}$ and ${ }_{(n)} \tilde{F}_{n}$ are stochastically comparable. Also, let ${ }_{(n)} Q$ be an arbitrary augmentation of an $n \times n$ north-west corner truncation of $Q$ and note that $\overline{{ }_{(n)} Q} \sqsubseteq{ }_{(n)} \tilde{Q}_{n}$, whence $\overline{{ }_{(n)} F} \sqsubseteq{ }_{(n)} \tilde{F}_{n}$. From the previous subsection, $\overline{{ }_{(n)} F} \sqsubseteq{ }_{(n+1)} \tilde{F}_{n+1} \sqsubseteq \tilde{F}$ for $n=0,1,2 \cdots$

Combining these, we obtain

$\overline{(n)}_{(n)}{ }_{(n)} F_{n} \sqsubseteq{ }_{(n)} \tilde{F}_{n} \sqsubseteq{ }_{(n+1)} \tilde{F}_{n+1} \sqsubseteq \tilde{F}$ which implies that ${ }_{(n)} \pi,{ }_{(n)} \pi_{n}{ }_{(n)} \tilde{\pi}_{n} \preceq_{(n+1)} \tilde{\pi}_{n+1} \preceq \tilde{\pi}$. Thus, the sequence $\left(_{(n)} \pi\right)$ is tight and ${ }_{(n)} \pi \rightarrow \pi$ componentwise as $n \rightarrow \infty$. Convergence in total variation follows in the same way as in the proof of Theorem 4.2 and the same is true of $\left.{ }_{(n)} \pi_{n}\right)$. Thus we have proved the following result.

Theorem 4.3 Let $Q$ be the $Q$-matrix of an irreducible Markov process which is dominated by a positive recurrent, stochastically monotone Markov process. Then, $\|(n) \pi-\pi\| \rightarrow 0$ as $n \rightarrow \infty$ where $\mathrm{p}$ is the unique invariant distribution for $Q$ and ${ }_{(n)} \pi$, for $n \in \mathbb{N}$, constitutes an invariant distribution of an arbitrary $n \times n$ north-west corner augmentation of $Q$.

As the augmentation ${ }_{(n)} Q_{n}$ is a special case of $\overline{{ }_{(n)} Q}$, it follows from the theorem that $\left\|_{(n)} \pi_{n}-\pi\right\| \rightarrow 0$ as $n \rightarrow \infty$. However, unlike the situation in which $F$ is stochastically monotone, it is not clear which of ${ }_{(n)} \pi$ and ${ }_{(n)} \pi_{n}$ provides the better approximation to $\pi$.

\subsection{Finitely Perturbed Stochastically Monotone Markov Processes}

Finally, we consider an even more general class of Markov processes which was introduced in [7]. We say that $Q$ is a finite perturbation of $\hat{Q}$ if the two $Q$-matrices differ in at most a finite number of columns. Let $\hat{Q}$ be stochastically monotone and suppose without loss of generality that $Q$ and $\hat{Q}$ differ in the first $k$ columns. Let $\tau: \min \{\hat{Q}(m): m=0,1, \cdots, k-1\}$ and construct a $Q$-matrix $\tilde{Q}$ as follows:

$$
\tilde{Q}(i, j)=\left\{\begin{array}{l}
-\tau, \text { if } j=i, 0 \leq i \leq k, \\
\tau+\sum_{m=0}^{k} \hat{Q}(i, m), \text { if } i<k, j=k \\
\sum_{m=0}^{k} \hat{Q}(i, m), \text { if } i \geq k, j=k \\
\hat{Q}(i, j), \text { if } j>k \\
0, \text { otherwise }
\end{array}\right.
$$

Observe that $\tilde{Q}$ is stochastically monotone. This is due firstly to the way in which the first k columns have been constructed from $Q$, and secondly to the agreement between the remaining columns of $Q$ with the corresponding columns of the stochastically monotone $\hat{Q}$. Now, $\tilde{Q}$ satisfies (17) and so, by Theorem 4.1, the minimal $Q$-function $F$ and $\tilde{Q}$-function $\tilde{F}$ are stochastically comparable. Direct application of Theorem 4.3 to $F$ and $\tilde{F}$ then yields the following result.

Theorem 4.4 Let $Q$ be a finite perturbation of a $Q$ matrix $\hat{Q}$ whose minimal $\hat{Q}$-function is irreducible, positive recurrent and stochastically monotone. Also, let $\mathrm{p}$ be the unique invariant distribution for $Q$ and denote the invariant distributions of arbitrary $n \times n$ north-west corner augmentations ${ }_{(n)} Q$ by ${ }_{(n)} \pi$. Then, $\|(n) \pi-\pi\| \rightarrow 0$ as $n \rightarrow \infty$

\subsection{Example}

Conrth-death process, whose tridiagonal $Q$-matrix

$$
Q(i, j)=\left\{\begin{array}{l}
\lambda_{i}, \text { if } i \geq 0, j=i+1, \\
\mu_{i}, \text { if } j \geq 0, i=j+1, \\
\lambda_{0}, \text { if } j=i=0, \\
-\left(\lambda_{i}+\mu_{i}\right), \text { if } j=i>0, \\
0, \text { otherwise, }
\end{array}\right.
$$

where $\left(\lambda_{i}, i \geq 0\right)$ are strictly positive birth rates and $\left(\mu_{i}, i \geq 1\right)$ are strictly positive death rates. Here, we take the state space $S$ to be the set of non-negative integers. Such processes can be used to model queues having memoriless arrival and service times, simple circuitswitched teletraffic networks and buffers in computer networks, etc.

Let $Q$ be the $Q$-matrix of an irreducible birth-death process. Then, it can be shown (see [1], Chapter 3) that $Q$ is regular if and only if $\sum_{i=0}^{\infty} \frac{1}{\lambda_{i} \tau_{i}} \sum_{j=0}^{i} \tau_{j}=\infty$

where $\tau_{0}=1$ and $\tau_{i}=\frac{\lambda_{0} \lambda_{1} \cdots \lambda_{i-1}}{\mu_{1} \mu_{2} \cdots \mu_{i}}$ for $i \geq 1$. Now for $Q$ regular, the unique minimal transition function $F$ is positive recurrent if and only if $A=\sum_{i=0}^{\infty} \frac{1}{\lambda_{i} \tau_{i}}=\infty$ and $B=\sum_{i=0}^{\infty} \tau_{i}<\infty$. The stationary distribution for $F$ is $\pi=(\pi(i), i \in S)$ where $\pi_{(i)}=\tau_{i} / B$

Now, it is straight forward to verify that $Q$ satisfies (17) and hence, by Theorem 4.1, $F$ is stochastically monotone. furthermore, by Theorem 4.2, we may use ${ }_{(n)} \pi_{n}$ to approximate $\pi$. Letting ${ }_{(n)} Q$ denote the north-west corner truncation on $\{0,1, \cdots, n\}$, augmenta- 
tion in column $n$ yields the matrix ${ }_{(n)} Q_{n}$ which differs from ${ }_{(n)} Q$ only in its $(n, n)$ element. More precisely, ${ }_{(n)} Q_{n}(n, n)=-\mu_{n}$ and ${ }_{(n)} Q_{n}(i, j)={ }_{(n)} Q(i, j)=Q(i, j)$, if either $i<n$ or $j<n$. The stationary distribution ${ }_{(n)} \pi_{n}$ corresponding to the augmentation ${ }_{(n)} q_{n}$ is given by ${ }_{(n)} \pi_{n}(i)=\tau_{i} / \sum_{j=0}^{n} \tau_{j}, 0 \leq i \leq n$ From this closed form expression, it can immediately be seen that ${ }_{(n)} \pi_{n}(i) \searrow \pi(i)$ as $n \rightarrow \infty$ since $\sum_{j=0}^{n} \tau_{j} \nearrow B<\infty$ as $n \rightarrow \infty$. Convergence in total variation then follows as in the proof of Theorem 4.2.

\section{Conclusion}

Here we have investigated procedures based on the augmentation of state-space truncations for approximating the stationary distributions of positive recurrent, continuous-time Markov processes on countably infinite state spaces. We have shown that approximation techniques first proposed for application to discrete-time markov chains are also efficacious in the continuous-time setting. Two classes of Markov process were considered: Exponentially ergodic processes and stochastically monotone processes. It was shown that the invariant distributions ${ }_{(\mathrm{n})} \pi$ corresponding to the augmented

$Q$-matrix $\overline{(\mathrm{n})} Q$ of finite statespace truncations of a

$Q$-matrix converge in total variation to the invariant distribution of the Markov process generated by that $Q$-matrix . It remains to study the speed of such convergence. An understanding of the convergence rate would enable the truncation size to be selected in order to guarantee that the measure ${ }_{(\mathrm{n})} \pi$ approximates $\pi$ to a desired degree of accuracy.

\section{Acknowledgements}

This work was supported by the Center for Mathematical Modeling (CMM) Basal CONICYT Program PFB 03 and FONDECYT grant 1070344. AGH would like to thank Servet Martinez for interesting discussion on the truncation of stochastically monotone processes. AGH dedicates this article to co-author Richard Tweedie, who passed away after this work was started.

\section{REFERENCES}

[1] W. Anderson, "Continuous-Time Markov Chains: An Applications-Oriented Approach,” Springer Series in Statistics, Springer-Verlag, New York, 1991.

[2] E. Seneta, "Finite Approximations to Infinite Nonnegative Matrices I," Proceedings of the Cambridge Philoso- phical Society, Vol. 63, No. 4, 1967, pp. 983-992. doi:10.1017/S0305004100042006

[3] E. Seneta, "Finite Approximations to Infinite Nonnega tive Matrices II: Refinements and Applications," Proceedings of the Cambridge Philosophical Society, Vol. 64, No. 2, 1968, pp. 465-470.

doi:10.1017/S0305004100043061

[4] E. Seneta, "Computing the Stationary Distribution for Infinite Markov Chains," Linear Algebra and Its Applications, Vol. 34, 1980, pp. 259-267. doi:10.1016/0024-3795(80)90168-8

[5] D. Gibson and E. Seneta, "Monotone Infinite Stochastic Matrices and Their Augmented Truncations," Stochastic Processes and Their Applications, Vol. 24, No. 2, 1987, pp. 287-292. doi:10.1016/0304-4149(87)90019-6

[6] D. Gibson and E. Seneta, "Augmented Truncations of Infinite Stochastic Matrices," Journal of Applied Probability, Vol. 24, No. 3, 1987, pp. 600-608. doi: $10.2307 / 3214092$

[7] R. Tweedie, "Truncation Approximations of Invariant Measures for Markov Chains," Journal of Applied Probability, Vol. 35, No. 3, 1998, pp. 517-536. doi:10.1239/jap/1032265201

[8] R. Tweedie, "Truncation Procedures for Nonnegative Matrices," Journal of Applied Probability, Vol. 8, No. 2, 1971, pp. 311-320. doi:10.2307/3211901

[9] R. Tweedie, "The Calculation of Limit Probabilities for Denumerable Markov Processes from Infinitesimal Properties," Journal of Applied Probability, Vol. 10, No. 1, 1973, pp. 84-99. doi:10.2307/3212497

[10] J. Kingman, "The Exponential Decay of Markov Transition Probabilities," Proceedings of the London Mathematical Society, Vol. 13, No. 1, 1963, pp. 337-358. doi:10.1112/plms/s3-13.1.337

[11] D. Down, S. Meyn and R. Tweedie, "Exponential and Uniform Ergodicity of Markov Processes," Annals of Probability, Vol. 23, No. 4, 1995, pp. 1671-1691. doi:10.1214/aop/1176987798

[12] R. Tweedie, "Criteria for Ergodicity, Exponential Ergodicity and Strong Ergodicity of Markov Processes," Journal of Applied Probability, Vol. 18, No. 1, 1981, pp. 122-130. doi: $10.2307 / 3213172$

[13] S. Meyn and R. Tweedie, "Markov Chains and Stochastic Stability," Springer-Verlag, London, 1993. doi:10.1007/978-1-4471-3267-7

[14] S. Meyn and R. Tweedie, "Computable Bounds for Convergence Rates of Markov Chains," Annals of Applied Probability, Vol. 4, No. 4, 1994, pp. 981-1011. doi:10.1214/aoap/1177004900

[15] B. Kirstein, "Monotonicity and Comparability of TimeHomogeneous Markov Processes with Discrete State Space," Statistics, Vol. 7, No. 1, 1976, pp. 151-168.

[16] A. Hart, "Quasistationary Distributions for ContinuousTime Markov Chains," Ph.D. thesis, University of Queensland, Brisbane, 1997.

[17] L. Breyer and A. Hart, "Approximations of Quasistationary Distributions for Markov Chains," Mathematical and 
Computer Modelling, Vol. 31, No. 10-12, 2000, pp. 69-79. doi:10.1016/S0895-7177(00)00073-X

[18] M. Kijima and E. Seneta, "Some Results for Quasista- tionary Distributions of Birth-Death Processes," Journal of Applied Probability, Vol. 28, No. 3, 1991, pp. 503-511. doi: $10.2307 / 3214486$ 